\title{
How to Talk About Incontinence: A Checklist
}

Claire Rosato-Scott (University of Leeds); Dr Dani J.

Barrington (The University of Western Australia); Dr Amita

Bhakta (Independent Consultant); Dr Sarah J. House

(Independent Consultant); Dr Islay Mactaggart and Jane

Wilbur (London School of Hygiene \& Tropical Medicine)

\section{0 institute of development} studies

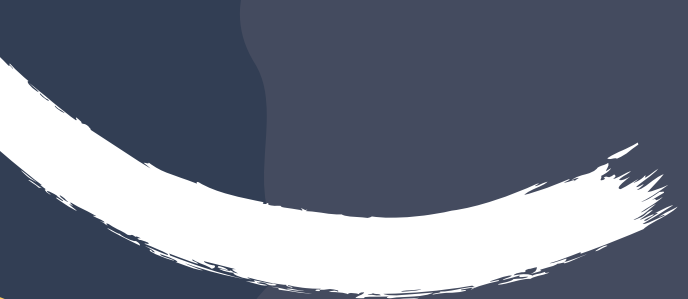




\section{How to Talk About Incontinence: A Checklist}

Incontinence is the medical term used to describe the involuntary loss of urine or faeces. Women, men, girls, boys and people of all genders, at any age, can experience incontinence. A person with incontinence can experience leakage occasionally, regularly or constantly; and leakage can happen at any time, day or night.

A person may also experience leakage of urinary or faecal matter due to not being able to get to the toilet in time or not wanting to use the toilet facilities available. This is known as social, or functional, incontinence.

In many low- and middle-income countries (LMICs) understanding of incontinence is still in its early stages: the term 'incontinence' may not be known, knowledge of the condition is rare, and the provision of support is lacking. Those who experience incontinence may face stigma due to having the condition, and this may affect their willingness or confidence to talk about it.

There is a need to better understand incontinence in LMICs, and how best to support people living with the condition to improve their quality of life. This requires having conversations with individuals that experience the condition, and with individuals who care for those who do: they will have the lived experiences of what it means to live with incontinence practically, emotionally and socially for them and their families.

Living with incontinence can have a range of impacts on the people living with it and their carers. These include increased stress and distress; additional needs for water and soap; and restricted ability to join in community activities, school or work. Living with incontinence can also lead to a range of protection issues. The potential challenges that people face may be quite diverse and may vary between people and households.

The checklist below, and corresponding page references to 'Incontinence: We Need to Talk About Leaks' can be used to increase your understanding of incontinence and the options available to support people living with the condition; and provide guidance on how to have conversations to understand how best to support people living with incontinence in your area. 


\section{CHECKLIST}

\section{DONE NOTES}

\section{a. INCREASE YOUR UNDERSTANDING OF}

INCONTINENCE

1. Learn about and understand:

- The different types of incontinence.

pg 7

- Who may experience incontinence.

- The impacts of experiencing incontinence (health and hygiene impacts / mental health, emotional and social impacts; practical and financial impacts; and protection risks).

- The options available to support people living with the condition (accessible water supply and sanitation facilities / sustainable supplies of basic hygiene items and facilities / products to aid the management of incontinence).

- Local attitudes about incontinence and practices for trying to manage it.

2. Practice explaining your understanding of incontinence in simple terms using culturally appropriate language:

- $\quad$ Ask local people living with it what words they think are respectful and clear, noting and respecting that this may vary between people. 


\section{CHECKLIST}

DONE NOTES

\section{b. TALK ABOUT INCONTINENCE TO UNDERSTAND HOW BEST TO SUPPORT PEOPLE LIVING \\ WITH INCONTINENCE IN YOUR AREA AND \\ ENCOURAGE CROSS-SECTORAL SUPPORT}

3. Engage with people that experience incontinence, or who care for people that do in your area

- Work with broader groups of people with characteristics that may make them more vulnerable to the condition, and who may also have knowledge of family, friends or neighbours living with the condition and /or

- Engage with specific actors who may have knowledge of people living with the condition to identify people living with incontinence; and/or

- Use strategies to encourage selfreferral, where it may be possible.

4. Talk about leaks. Incontinence is a topic that is best suited to in-depth discussions, which are flexible in structure and guided by the participant:

- Always obtain informed consent from the interviewee before beginning any discussions.

- Ensure that you have pre-prepared the contact details of referral services that may be required if you meet someone facing particular challenges (such as health, disability or protection related services; or WASH actors who could respond to particular needs).

- Ensure that the environment is as comfortable and private as possible (it should be somewhere where other people cannot overhear; where the people speaking feel safe to speak on sensitive issues; when possible have men speaking with men and women speaking with women; and check if the interviewee would like to have anyone specifically present or absent). 


\section{CHECKLIST}

DONE NOTES

- Be an empathic interviewer (be confident and do not show embarrassment; put the person at ease and build trust by being friendly and building rapport before asking questions on incontinence; and use active listening skills).

- Consider if it would be useful to take some simple options for support or to use as discussion aids to get feedback on what might be most useful (such as soap, reusable underwear, mattress protectors etc.). Note that these should be offered discretely so as to not cause additional embarrassment, and only if the opportunity for the person to have a sustainable supply has been considered.

- Make efforts wherever possible to ensure that the people you speak with and learn from receive feedback on what came out of the discussions and learning: realising that you were interested and listened, and that they are being part of the solution through sharing their difficult experiences with you for you to learn from is likely to be motivational for many people living with this condition on a daily basis.

\section{Advocate to de-mystify and de- stigmatise incontinence}

- Normalise incontinence in conversations, guidance documents and other publications.

pg 24

- Encourage engagement with, and by, and provision of support from other sectors, including Health (including Nutrition and Occupational Therapists), Protection, Gender-Based Violence, Disability and Older Persons, Midwifery, Children, Gender, Livelihoods and WASH.

- Document what you learn on working with people with incontinence, challenges, how you have worked out to respond to them, and good practices. Share widely as learning is still in its early stages, taking care to keep details anonymous and asking permission for sharing specific case studies. 
Correct citation: Rosato-Scott, C., Barrington, D.J., Bhakta, A., House, S.J., Mactaggart, I. and Wilbur, J. (2020) How to Talk About Incontinence: A Checklist, The Sanitation Learning Hub Brighton: IDS, DOI: 10.19088/SLH.2020.006

First published in 2020

(c) Institute of Development Studies 2020

Some rights reserved - see copyright license for details.

\section{ISBN 978-1-78118-708-1}

This pubication is licensed under the Creative Commons Attribution-Non-Commercial-NoDerivs 3.0 Unported License (https://creativecommons.org/licenses/by-nc-nd/3.0/).

Attribution: You must attribute the work in the manner specified by the author or licensor.

Non-commercial: You may not use this work for commercial purposes No Derivative Works: You may not alter, transfer, or build on this work.

Users are welcome to copy, distribute, display, translate or perform this work without written permission. For any reuse or distribution, you must make clear to others the licence terms of this work. If you use the work, we ask that you reference the SLH website and send a copy of the work or a link to its use online to the following address: Sanitation Learning Hub, Institute of Development Studies, University of Sussex, Brighton, BN1 9RE, UK (SLH@ids.ac.uk).

\section{(c) creative}

This document has been financed by the Swedish International Development Cooperation Agency, Sida. Sida does not necessarily share the views expressed in this material. Responsibility for its contents rests entirely with the authors.

IDS, a charitable company limited by guarantee: Registered Charity No.

306371; Registered in England 877338; VAT No. GB 350899914

f /SanLearningHub

(7) @SanLearningHub

(0) @SanLearningHub

\section{Find out more}

Subscribe to the SLH newsletter, share your experiences and

contribute to the SLH website by visiting

https://sanitationlearninghub.org/connect-share-learn/ 